\title{
Ancestral cancer genes shaping evo-devo: An integrated biochemical and computational approach (Review)
}

\author{
DIMITRIOS VLACHAKIS ${ }^{1-3}$, ELENI PAPAKONSTANTINOU ${ }^{1}$, ASPASIA EFTHIMIADOU ${ }^{4}$,

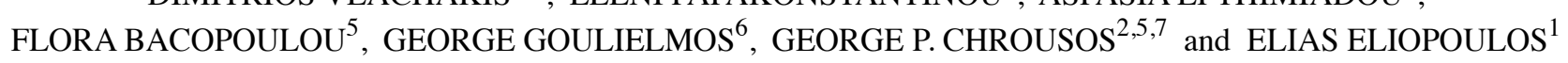

${ }^{1}$ Genetics and Computational Biology Group, Laboratory of Genetics, Department of Biotechnology, Agricultural University of Athens, 11855 Athens; ${ }^{2}$ Division of Endocrinology and Metabolism, Center of Clinical, Experimental Surgery and Translational Research, Biomedical Research Foundation of The Academy of Athens, 11527 Athens, Greece; ${ }^{3}$ Department of Informatics, Faculty of Natural and Mathematical Sciences, King's College London, London WC2R 2LS, UK; ${ }^{4}$ Hellenic Agricultural Organization-Demeter, Institute of Soil and Water Resources, Department of Soil Science of Athens, 13561 Athens; ${ }^{5}$ Division of Endocrinology, Metabolism and Diabetes,

First Department of Pediatrics, National and Kapodistrian University of Athens Medical School, 'Aghia Sophia' Children's Hospital, 11527 Athens; ${ }^{6}$ Section of Molecular Pathology and Human Genetics, Department of Internal Medicine, School of Medicine, University of Crete, 71003 Heraklion;

${ }^{7}$ UNESCO Chair on Adolescent Health Care and University Research Institute of Maternal and Child Health and Precision Medicine, Medical School, National and Kapodistrian University of Athens, 11527 Athens, Greece

Received January 14, 2020; Accepted April 21, 2020

DOI:10.3892/wasj.2020.48

\begin{abstract}
Evo-devo has been shaped by a myriad of natural forces, such as environmental factors, opportunism, survival of the fittest and even disease, including cancer. The influence of the environment and evolutionary stress through natural selection has been driving life since the very beginning. However, it is not only through the development of jaws and claws that species survive and evolve. Disease is just another tool in nature's arsenal of evolution. A repertoire of gene-coding and non-coding regions, as researchers recently begin to understand, play a pivotal role in diseases, including cancer. The present review focuses on elucidating the role of signaling, metabolism and transcription processes as stake holders of evolutionary and developmental biology, namely evo-devo, under the prism of reduced survival and disease through cancer genes. The mechanisms through which ancestral cancer genes can shape evolution and development, when 'cancer' is viewed as a robust, evolvable system, are discussed, focusing on its evolutionary origins as an alternative approach.
\end{abstract}

Correspondence to: Professor Dimitrios Vlachakis, Genetics and Computational Biology Group, Laboratory of Genetics, Department of Biotechnology, Agricultural University of Athens, 75 Iera Odos, 11855 Athens, Greece

E-mail:dimvl@aua.gr

Key words: evo-devo, ancestral genes, cancer, signaling, metabolism, transcription

\section{Contents}

1. Introduction

2. Signaling in cancer

3. Metabolism in cancer

4. Transcription in cancer

5. Computational methods for pharmacological targeting in cancer

6. Conclusions

\section{Introduction}

Evolution can be viewed as the accidental result of random mutations, in both gene-coding and non-coding regions of the DNA, leading to the amazing diversity of life forms currently observed on earth. Still, it is the genetic, epigenetic, epitranscriptomic and socio-environmental events that act upon the developmental mechanisms, that guide evolution and dictate the survival of a single living unit and a species' reproductive success across and along generations. Genetic alterations that can 'switch off' genes or enhance their expression, or even relegate them to non-coding segments of DNA, are merely part of the equation. Genetic and environmental challenges are determining evolution in terms of the survival of the fittest. Natural selection guides the evolutionary process by favoring genes for their ability to propagate their information through generations.

Evolutionary and developmental biology, namely evo-devo, refers to the mechanisms through which developmental processes determine and modulate variations, and dictate the evolutionary outcomes throughout the course of history. The main focus of evo-devo, as previously described 
by Hendrikse et al, is the capacity of developmental systems for adaptive evolution through these variations, or else evolvability (1). Robustness, that is the ability of a system to be resistant to change, is a basic concept of evolvability. Robust organisms have the ability to accumulate genetic variations with no effects on their phenotype; however, this cryptic evolutionary potential of their genomes can be released in a new environmental and genetic background, enhancing the probability of the organisms to adapt (2).

Cancer can be viewed as a robust, evolvable system, and focusing on the evolutionary origins of cancer can be used as an alternative approach (3). The genes that are responsible for the processes of proliferation inhibition, cell death, division of labor, resource allocation and extracellular environment maintenance, are the genes that sustain the viability of complex multicellular organisms (4). They are also the genes that malfunction in cancer (5). A wide number of oncogenes and tumor suppressors have been discovered over the past 50 years, and almost all cancers are driven by genetic alterations in these genes. Hence, the genetic basis of cancer has an ancient past. According to the atavistic theory, cancer is the result of the accumulation of mutations that reprogram the cell into adopting a primitive phenotype, by reactivating an ancient behavior characterized by highly conserved survival (6). This phenotype is characterized by the upregulation of ancestral genes with unicellular evolutionary origins, disrupting the genetic regulatory network that rules upon complex multicellular organisms and leading to uncontrolled proliferation in adverse environmental conditions (7-9).

As such, cancer events can be considered as a drawback or a side-effect of evolution. However, under the scope of evo-devo as an interplay of yin and yang, it can be argued that cancer events can be viewed as an integral component of evolution (10). At a (cancer) cell-level, this phenotype depicts a 'drive for survival' with its evolutionary roots in the early transition stages from unicellularity to multicellularity that lead to a diversity burst, whereas at the (host) organism-level, a cancer event outlines the 'unfitness' of the host for natural selection. The trading between two evolutionary states, an ancestral one characterized by stochasticity and plasticity/flexibility with a high evolutionary rate and a recent, synchronous equilibrium with a refined regulatory machinery (however threatened by a variety of stressors), by activating and deactivating 'ancient' genes may be the gear which sets in motion the forces that formulate life by survival selection and thus, shaping evo-devo.

In the following sections, focus is paid on elucidating the role of ancestral genes involved in signaling, metabolism and transcription pathways as stake holders of evo-devo under the prism of reduced survival and disease through 'cancer'. Collectively, a list of inhouse curated genes that are involved in signaling, metabolism and transcription in cancer, by harvesting the NCBI Gene Database (11) with the respective keywords and filtering out the noise, is provided in Table SI. Notably, as shown in Fig. 1, 44.58\% of cancer-associated genes are involved in transcription pathways, whereas the co-occurrence of signaling- and metabolism-associated genes in cancer is 17.07 and $17.35 \%$, respectively.

\section{Signaling in cancer}

Cells are able to respond and adapt to their environment through signal transduction. Signaling pathways coordinate intra- and intercell communication, as well as the communication between cells and the extracellular matrix. Genetic and epigenetic alterations often lead to the disruption of signaling networks and enable cancer cells to escape regular control mechanisms (12). These alterations are involved in cancer progression, such as cell growth and proliferation, angiogenesis and inflammation. Oncogenic mutations can cause the hyperactivation of the signaling pathways and the overexpression of the affected genes or produce mutated proteins with deficient activity, and deletions or other mutations can inactivate tumor suppressors that normally act as negative regulators in signaling (13). Even though the mutational profile of tumors is highly diverse, there are a small number of mutations with a causative role in oncogenesis, termed 'drivers', that lead the cancer cell to a fitness advantage $(14,15)$. 'Passengers' on the other hand are estimated to account for $\sim 97 \%$ of mutations in cancer and are mostly considered random non-functional mutations, although there are indications of a certain fitness cost for the tumor upon elevated passengers load $(16,17)$. Driver mutations have been shown to affect a limited number of signaling pathways that regulate three core cellular processes: Cell fate, cell survival and genome maintenance, rendering the focus towards a consensus of mutated driver pathways rather than individual driver genes (18-20).

A representative example of driver mutation is p53 tumor suppressor protein, that is a crucial component in the regulation of cell cycle in multicellular organisms (21). The evolutionary history of the p53 pathway can be traced back to the beginning of multicellularity and is activated upon cellular stress signals $(22,23)$. The previous evolutionary study by Belyi et al traced the origins of the p53 family genes back to the unicellular choanoflagellates and the early metazoan sea anemone, where the ancestral gene was found to be related to the p63/p73-like gene, and upon gene duplication in the early vertebrate lineage, the resulting product was closely associated with that of the p53 gene (24). The p53 protein is a transcription factor that binds to specific DNA sequences and acts as an anticancer promoting agent, and is therefore also known as the 'genome guardian' (25). It activates DNA-repair proteins, induces growth arrest and initiates apoptosis. p53 is encoded by the TP53 gene, which is the most frequently mutated gene in human cancers. In almost half of human cancer types, p53 is inactivated by mutation, leading to a severely reduced tumor suppressive activity (26).

Another example of genetic alterations deregulating the signal transduction and promoting tumor progression is the phosphoinositide 3-kinase (PI3K)/Akt signaling pathway. The PI3K/Akt pathway is evolutionary conserved and regulates metabolism, proliferation, cell survival, growth, motility and apoptosis in response to extracellular signals (27). Both PI3K and Akt enzymes have a wide phylogenetic distribution and have been identified in unicellular organisms and have later evolved through complex duplication patterns $(28,29)$. Activating mutations in PI3K, Akt and PIK3R1 have been described in cancer and result in persistent amplification of the PI3K/Akt pathway and aberrant cell proliferation. 


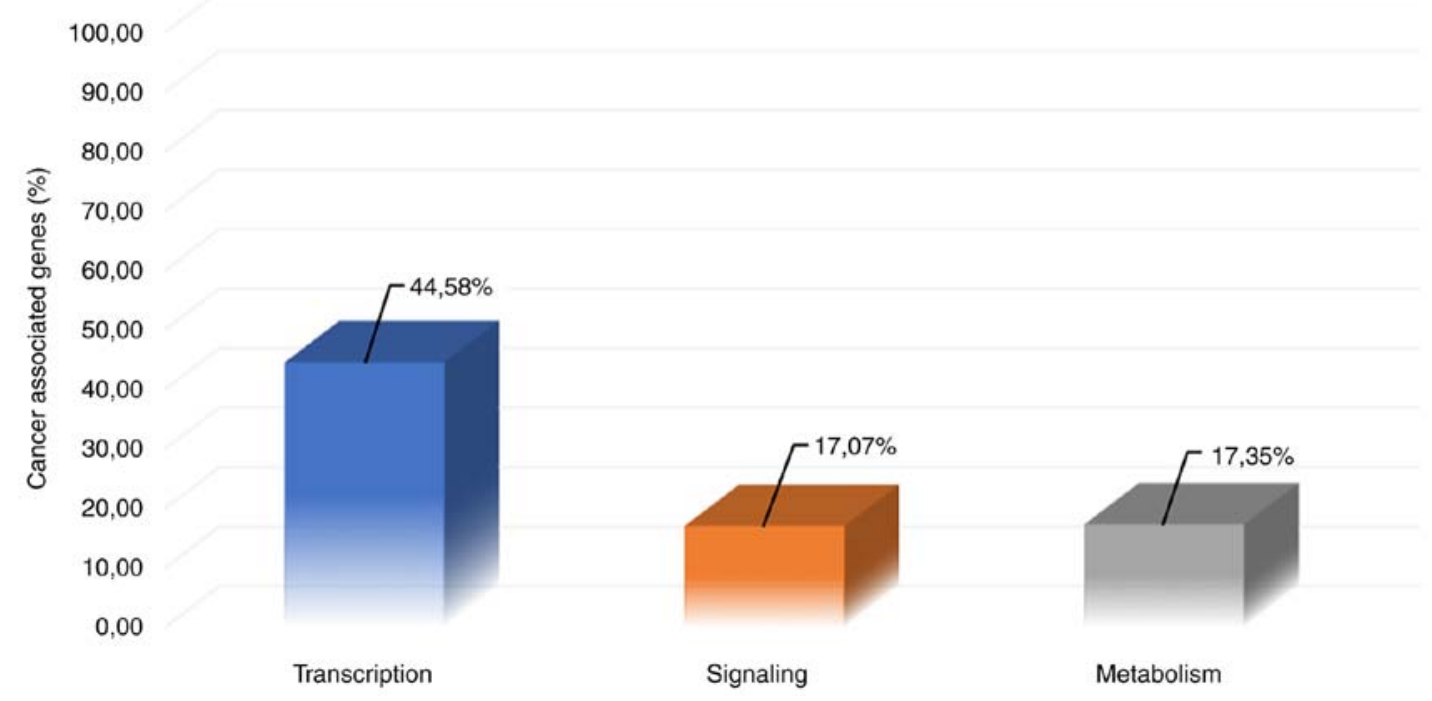

Figure 1. Percentage of transcription, signaling and metabolism associated genes on the total number of cancer-associated genes.

Inactivating mutations and deletions of phosphatase and tensin homolog deleted on chromosome 10 (PTEN) and inositol polyphosphate-4-phosphatase, type II (INPP4B) tumor suppressors lead to unrestrained downstream signaling (30). Mutations in TSC1 and TSC2 tumor suppressors hyperactivate signaling by mammalian target of rapamycin (mTOR)C1, a crucial target of the PI3K/Akt pathway $(31,32)$.

The Notch signaling pathway is involved in cell development and differentiation, and has also been found to be aberrantly activated in a number of different solid tumors through mutations (33). The Notch pathway is based on cell-cell contacts for signal transduction and is highly involved in tumor metastasis $(34,35)$. Of note, both gain- and loss-of-function mutations in NOTCH isoforms are described, reverting the ability of the Notch signaling pathway from an oncogene to a tumor-suppressor in a highly context-dependent manner $(36,37)$.

A gene that is present in unicellular organisms and associated with cancer in multicellular organisms is the helicase-associated endonuclease for fork-structured DNA (Hef). Hef is a protein found in Archaea and is required for the processing of blocked replication forks. The vertebrate Hef ortholog appears to participate in the Fanconi anemia-related (FA) tumor suppressor pathway, while the archaebacterial Hef processes stalls replication forks in order for them to be repaired by homologous recombination (38). Vertebrate Hef seems to also play a role in DNA repair mechanisms, where it interacts directly with DNA structures that are DNA replication intermediates and may contribute to resolving DNA crosslinks through a complex association with the FA complementation group $\mathrm{C}$ gene (38).

The 70-kDa family of heat shock proteins (HSP70) is regarded as one of the most conserved groups of proteins in evolution. The majority of organisms include multiple members of the protein, while some, such as archaebacteria, include at least one (39). Hsp70 proteins are molecular chaperones that participate in a diverse group of processes, including protein folding and remodeling as they act virtually at all stages of protein life, from synthesis to degradation, and thus are essential in protein homeostasis (40). Cancer cells rely heavily on the mechanisms of HSP70s regulation for survival. The majority of human tumors, as an example, overexpress HSP70 family members, and this overexpression can be used as a biomarker for a poor prognosis (39). This overexpression in the case of mammary carcinoma may be largely due to the proliferation of misfolded proteins and overexpressed oncoproteins that trigger the transcription of HSP genes. A prime example is the ability of Tp53 to suppress the HSP70 promoter, and the loss of this protein, a common event in cancer, may lead to an increase in the expression of said chaperone proteins (41).

\section{Metabolism in cancer}

Metabolic reprogramming is a hallmark of cancer. The activation of cancer genes and the progression of a cancer event alter the metabolic process of the cell, in order for it can meet the high demands of cancer cells in energy and nutrient resources. The majority of malignant cells switch to aerobic glycolysis as a preferred metabolic pathway, a phenomenon also known as the Warburg effect, producing a high amount of secreted lactate $(42,43)$. The acquired glycolytic behavior of cancer cells holds for the sustainability of their bioenergetics, biosynthesis and redox demands. Even though oxidative phosphorylation is a much more efficient source of ATP compared to glycolysis, it has been shown that the high glycolytic rates in cancer cells are favored due to the increased levels of precursor metabolites for anabolic pathways (44). In addition, glycolysis enables cancer cells to deal with unfavorable conditions, such as hypoxia and a low nutrient supply, and to adapt to their highly heterogeneous microenvironment, thus maintaining an evolutionary advantage (45).

The activation of specific oncogenes and the loss of tumor suppressors, along with the upregulation of the PI3K pathway is controlling the metabolic switch in cancer $(46,47)$. The activation of Myc, Ras and Akt, and the inactivation of p53 have been shown to upregulate glycolytic enzymes and glucose transporters and stimulate glycolysis $(48,49)$. Additionally, to maintain this glycolytic phenotype, cancer cells upregulate a 
number of plasma membrane transporters, such as monocarboxylate transporters (MCTs), that mediate the proton-linked transport of metabolic monocarboxylic acid (50). Specifically, MCT1 and MCT4 isoforms are highly involved in maintaining the metabolic phenotype of cancer cells by facilitating the transport of lactate across the plasma membrane and regulating the intracellular $\mathrm{pH}$ by co-transporting a proton (51). MCT4 has the lowest affinity for lactate among MCTs and is the main isoform that mediates lactic acid efflux from glycolytic cells, including white skeletal muscle fibers, astrocytes, immune cells, chondrocytes and hypoxic cells (52). MCT4 expression is upregulated under hypoxic conditions and oxidative stress by hypoxia-inducible factor $1 \alpha$ (HIF-1 $\alpha)$, favoring lactate extrusion from the cell MCTs and preventing intracellular acidification, thus promoting a number of carcinogenic processes (53-55). MCT4 is also recognized for its role in metastasis and is shown to be upregulated in the tumor stroma by oncogenes Ras and nuclear factor (NF)- $\kappa \mathrm{B}(56)$. The increased expression of MCT4 and its ancillary protein, CD147, is also associated with a poor prognosis in a number of types of cancer (57). c-Myc has been shown to increase the expression of MCT1, as in with pyruvate dehydrogenase kinase-1 (PDK-1) that phosphorylates the pyruvate dehydrogenase enzyme and lactate dehydrogenase A ( $\mathrm{LDH}-\mathrm{A})$, an enzyme that catalyzes the conversion of lactate to pyruvate $(58,59)$. As such, MCTs are considered as promising therapeutic targets for disrupting the glycolytic cascade of cancer cells.

One of the key enzymes found in all organisms and a key component in the metabolic reprogramming of cancer, is glutamine synthetase (GS). GSs is responsible for nitrogen metabolism, where it participates in the biochemical reaction of ammonia assimilation and in glutamine biosynthesis. It has been demonstrated that GS expression is induced by the Myc oncogene, resulting in increased glutamine anabolism that is associated with increased cell proliferation, survival and transplant tumor growth. All of the above lead to the conclusion that GS expression plays an important role in Myc-driven carcinogenesis (60).

Three types of GS have been reported from previous studies. The two most basic types are GSI and GSII. GSI has been identified in prokaryotic organisms, whereas GSII has been identified mainly in eukaryotes, but also in some prokaryotes belonging to the families Rhizobiaceae, Frankiaceae and Streptomycetaceae (61). Finally, the third type of GS is GSIII, which has been observed in the anaerobes Bacteroides fragilis (62) and Butyrivibrio fibrisolvens (63). These types differ in both their primary and tertiary structure. GSI consists of 12 identical subunits, which are structured in 2 layers, each consisting of 6 subunits. At the active site, the synthetase contains a pair of $\mathrm{Mn}^{++}$ions and forms 2 antisense $\pi$-structures, one in the carboxyl terminal end of a subunit and the other in the N-terminal end of the adjacent subunit. GSII and GSIII contain fewer subunits than GSI, with GSII consisting of 8 subunits and GSIII of 6 . It is still worth noting that a fourth type of synthetase has been identified in Rhizobium leguminosarum and is reported as glnT, which has more common elements with prokaryotic GSI (64).

According to the results of previous evolutionary analyses in which the genes encoding GS were aligned in different organisms, it was found that the genes of GSI and GSII existed
1,700 million years prior to the divergence of prokaryotes and eukaryotes (60). In the study by Shatters and Kahn, it was denoted that the common ancestor of the GSII genes in Rhizobiaceae and the host plant was older than the plant itself (65). Moreover, it is surprising that the genes encoding the rice and pea chloroplast GSII enzymes are more closely related than the corresponding genes of the same species. Finally, it is estimated that mitochondrial GSII is 1,050 million years old (60).

\section{Transcription in cancer}

Dysregulation in the gene expression program is also a signature in cancer. Transcriptional regulation and gene expression are controlled by a vast number of transcription enzymes, transcription factors, co-factors and chromatin regulators that are interacting in a highly coordinated manner and perturbations on the transcriptional mechanism controls are evident in all tumors (66). Gene alterations that result in the activation of oncogenes, the inactivation of tumor suppressors and the upregulation of protein kinases can promote transcription and subsequently drive cell proliferation.

RNA polymerases are highly conserved in evolution, and their subunits exhibit a common structural framework, while being operated by closely related molecular mechanisms (67). In their study, Werner and Grohmann (67) denoted that the last universal common ancestor of bacteria, Archaea, and Eukarya carried an RNA polymerase very similar to the simplest form of contemporary RNAPs found in bacteria, while Shin et al indicated that archaeal RNAPs share more properties with their eukaryotic homologs (68). RNA polymerase I (Pol I) is the most highly engaged enzyme in the general transcriptional machinery, accounting for $>60 \%$ of the overall cell transcriptional activity (69). Cancer cells have a higher biosynthetic demand, exhibiting upregulated ribosome biogenesis, known as 'nucleolar hypertrophy', to support cell growth and uncontrolled cell proliferation. Ribosome production is strictly dependent on the Pol I transcription machinery in the nucleolus; thus, Pol I activity is further increased in proliferating cells (70). A number of oncogenic factors driving accelerated Pol I transcription have been reported over the years. The oncogenic activity of Myc has been shown to stimulate Pol I transcription and enhance ribosomal biogenesis (71,72). Activated mTOR induces Pol I transcription and ribosome synthesis by activating upstream binding factor (UBF) and transcription initiation factor 1A (TIF1A) (73). The inactivation of PTEN tumor suppressor results in the constitutive activation of the oncogenic PI3K/AKT pathway and tumorigenesis (74). A number of tumor cells harbor mutations that affect both pRb and p53 tumor suppressors, that normally suppress Pol I transcription and inhibit cellular rRNA synthesis, having an added impact on Pol I activity (75).

Cancer cells also exhibit an upregulated activity of RNA polymerase II (Pol II) to produce a high number of transcripts, including oncogenes and anti-apoptotic factors, supporting rapid growth and resistance to apoptosis (76). The control of Pol II is highly regulated by transcriptional factors and non-coding RNAs (ncRNAs) and is critical for the cell homeostasis. Genetic variations, such as mutations in transcription factors that control Pol II can result in a disruption 
of the pause release and elongation process. Increased levels of c-Myc cause transcriptional amplification by accumulating at promoters regions and producing high levels of transcripts, thus inducing tumorigenesis (77). Gene fusion events have been shown to alter the transcription elongation, as in the case of the chromatin regulator MLL in leukemias (78). Long non-coding RNAs have also been implicated for their role in cancer progression, functioning as transcriptional regulators. The lncRNA ANRIL induces the transcriptional repression of members of the INK4A/ARF/INK4B locus, which encode tumor suppressors whose deactivation is associated with various types of cancer (79).

\section{Computational methods for pharmacological targeting in cancer}

Developing efficient therapeutic methods for anticancer drug design remains a challenge, even though success stories have been reported over the past years. Cancer is a heterogeneous disease and a deeper understanding of the underlying molecular mechanisms that drive its initiation, progression and metastasis is crucial for providing effective treatment and improved diagnostics. Through the advances of bioinformatics and omics technologies, a wide variety of computational methods can be applied for pharmacological targeting in cancer.

Structure and ligand-based drug design are principal methodologies for drug discovery and lead optimization. Ligand-based approaches use the information of known active and/or inactive molecules to generate SAR models, whereas structure-based approaches use the structural information of the protein target to discover lead molecules as potent inhibitors (80). Homology modelling techniques are applied when an experimentally determined structure of a protein is not available. Molecular docking and molecular dynamics are extensively used to simulate the conformational state of a protein target and protein-ligand interactions to estimate the binding affinity. Virtual high-throughput screening through docking of small molecule libraries have been successfully applied for the identification of novel inhibitors with anticancer properties, as in the case of targeting Cdc25A phosphatases and protein kinase CK2 $(81,82)$. The physicochemical properties of small molecules with potent activity can be also analyzed through statistical methods and the applications of artificial intelligence (AI), such as machine learning, in the pursuit of a selective inhibitor. A key factor for the success of these strategies is to target specific biomolecules involved in functional molecular and biological traits that distinguish cancer cells from normal cells, known as the hallmarks of cancer.

Omics data are accumulating rapidly and can be assessed through statistical analysis and computational methods to uncover potential targets for efficacious therapeutics in cancer. Systems biology approaches, including computational modeling, network analysis, gene signature analysis, functional genomics, protein-protein interactions and high-throughput screening, are efficient tools for advancing the prediction of efficient therapeutics in complex diseases, such as cancer (83). In network analysis, computational models of signaling networks are designed and used to predict systems properties that can indicate and prioritize protein targets for cancer therapy (84). Computational approaches have been used for the prediction of functional impact of mutations and discriminate driver from passenger mutations, based on evolutionary conservation, protein structure modifications and observed recurrence in existing cancer datasets (85). Chen et al illustrated the oncogenic signatures in the tyrosine kinase family through an evolutionary analysis concluding that gain-of-function mutations are causing reverse evolution on the oncogenes supporting the cancer atavistic model (86). An interesting computational analysis in gene expression data was previously presented by Trigos et al (87), where 7 solid tumors were investigated with regards to their corresponding gene ages using phylostratigraphy. The results indicated that a common feature in tumors was a trend for the preferential expression of more ancient genes. The authors reported that these cellular processes assigned to a unicellularity origin were more active in tumors. Additional research demonstrates the effectiveness of evolutionary network analysis to identify prognostic cancer modules (88). These results provide a completely new perspective in identifying suitable pharmacological targets based on the evolutionary age of their encoding genes.

\section{Conclusions}

Cancer is a complex disease and its underlying mechanisms have yet to be fully elucidated. However, critical nodes can be identified under the scope of its evolutionary origins. The emergence of long-living multicellular animals demands the evolution of mechanisms that operate on various levels (including on individual cells, tissue organization, and the whole body) in order to maintain an appropriate number of cells within a specific tissue and limit cancer growth (89).

Another aspect of the evolutionary link of cancer to unicellularity concerns lateral gene transfer between bacteria and eukaryotes, particularly when symbiotic relationships are present (90). Given that human somatic cells are in a state of coexistence with various kinds of bacteria, the integration of bacterial genetic material is hypothesized to disrupt tumor suppressor genes or proto-oncogenes, acting as a mutagen, or the possibility for integrated bacterial gene to be transcribed by the mechanisms of the recipient human cell, leading to the production of a polypeptide with unpredictable repercussions on the cell (91). In a 2017 study, the tissues of patients suffering from esophageal cancer were analyzed, affirming the presence of the bacterium Fusobacterium nucleatum. Through microarray analysis, it was found that the presence of the bacterium affected cellular pathways in the cancer tissues, namely the cytokine-to-cytokine receptor interaction. These findings suggested that the presence of the bacterium in the cells induced the activation of chemokines, such as CCL20, contributing to tumorigenesis (92). Other researchers have begun to elucidate the effects of bacteria-human lateral gene transfer on the development of various cancer types. Bacterial DNA integrations have been found in human mitochondrial genome and more specifically, in samples of acute myeloid leukemia, identifying bacterial integration in genes known to be upregulated in stomach adenocarcinoma, an integration that appeared to take place in the 5'-UTR and 3'-UTR of those proto-oncogenes (93).

The regulatory network of living systems has been finely tuned through evolution and genetic and environmental perturbations can compromise the viability equilibrium of the cell and 
ancestral genes that are dysregulated can be encountered in all the critical cell signaling pathways. Upon tumorigenesis, uncontrolled proliferation and metastasis, a number of properties of multicellular organisms are dysregulated or lost (94). Genes originating from unicellular ancestors are either specifically activated or required for maintenance of cancer phenotype (95). It is argued herein that these ancestral cancer genes represent an integral part of evolution, by disrupting the acquired balance of the multicellular organisms and driving disease through cancer as a means for change and evolvability.

Cancer itself may be considered as an evolutionary system, in which cancer cells acquire mutations that allow them to survive, compete for space and resources, evade the immune system, and even cooperate in order to disperse and colonize new organs (96). Several factors, from radiation to chemicals to aging, can promote the evolution of cancer by increasing mutation frequency and promoting the selection of adaptive mutations. In direct correspondence with animal evolution, cancer cells respond to environmental adversities by selecting the clone which is most fit for survival. Therefore, it appears that cancer and tumor-suppressive mechanisms are engaged in an evolutionary arms race with each other (10). Considering this, the evolutionary aspect of cancer may help to predict the response of cancers to drugs and therapy, and lay the foundation for optimal treatment with immunotherapy, drugs, or chemotherapy.

\section{Acknowledgements}

Not applicable.

\section{Funding}

DV would like to acknowledge funding from: i) Microsoft Azure for Genomics Research Grant (CRM:0740983); ii) FrailSafe Project (H2020-PHC-21-2015-690140) 'Sensing and predictive treatment of frailty and associated co-morbidities using advanced personalized models and advanced interventions', co-funded by the European Commission under the Horizon 2020 research and innovation program; iii) Amazon Web Services Cloud for Genomics Research Grant (309211522729); iv) AdjustEBOVGP-Dx (RIA2018EF-2081): Biochemical Adjustments of native EBOV Glycoprotein in Patient Sample to Unmask target Epitopes for Rapid Diagnostic Testing. A European and Developing Countries Clinical Trials Partnership (EDCTP2) under the Horizon 2020 'Research and Innovation Actions' DESCA. EE would like to acknowledge funding by the project 'INSPIRED-The National Research Infrastructures on Integrated Structural Biology, Drug Screening Efforts and Drug Target Functional Characterization' (Grant MIS 5002550) and by the project: 'OPENSCREEN-GR An Open-Access Research Infrastructure of Chemical Biology and Target-Based Screening Technologies for Human and Animal Health, Agriculture and the Environment' (Grant MIS 5002691), which are implemented under the Action 'Reinforcement of the Research and Innovation Infrastructure', funded by the Operational Programme 'Competitiveness, Entrepreneurship and Innovation' (NSRF 2014-2020) and co-financed by Greece and the European Union (European Regional Development Fund). EP would like to acknowledge funding by the State Scholarships Foundation (IKY)-European Union (European Social Fund-ESF) and Greek national funds through the action entitled 'Strengthening Human Resources Research Potential via Doctorate Research' in the framework of the Operational Program 'Human Resources Development Program, Education and Lifelong Learning' of the National Strategic Reference Framework (NSRF) 2014-2020.

\section{Availability of data and materials}

A list of Homo sapiens genes associated with cancer, signaling, metabolism and transcription as downloaded by the NCBI Gene Database (66) are reported and their occurrence in cancer, signaling, metabolism and transcription databases are colour coded. All data generated or analyzed during this study are included in this published article or are available from the corresponding author on reasonable request.

\section{Authors' contributions}

DV, EP, AE, FB, GG, GPC, EE all equally contributed to the writing, drafting, revising, editing, reviewing, and the conception and design of the study. All authors have read and approved the final manuscript.

\section{Ethics approval and consent to participate}

Not applicable.

\section{Patient consent for publication}

Not applicable.

\section{Competing interests}

The authors declare that they have no competing interests.

\section{References}

1. Hendrikse JL, Parsons TE and Hallgrímsson B: Evolvability as the proper focus of evolutionary developmental biology. Evol Dev 9: 393-401, 2007.

2. Pavlicev M and Wagner GP: Coming to Grips with evolvability. Evolution: Educ Outreach 5: 231-244, 2012.

3. Tian T, Olson S, Whitacre JM and Harding A: The origins of cancer robustness and evolvability. Integr Biol (Camb) 3: 17-30, 2011.

4. Aktipis CA, Boddy AM, Jansen G, Hibner U, Hochberg ME, Maley CC and Wilkinson GS: Cancer across the tree of life: Cooperation and cheating in multicellularity. Philos Trans R Soc Lond B Biol Sci 370: pii: 20140219, 2015.

5. Hanahan D and Weinberg RA: Hallmarks of cancer: The next generation. Cell 144: 646-674, 2011.

6. Bussey KJ, Cisneros LH, Lineweaver $\mathrm{CH}$ and Davies PCW: Ancestral gene regulatory networks drive cancer. Proc Natl Acad Sci USA 114: 6160-6162, 2017.

7. Chu XY, Jiang LH, Zhou XH, Cui ZJ and Zhang HY: Evolutionary origins of cancer driver genes and implications for cancer prognosis. Genes (Basel) 8: pii: E182, 2017.

8. Chen $\mathrm{H}$, Lin F, Xing $\mathrm{K}$ and He X: The reverse evolution from multicellularity to unicellularity during carcinogenesis. Nat Comm 6: 6367, 2015

9. Trigos AS, Pearson RB, Papenfuss AT and Goode DL: Somatic mutations in early metazoan genes disrupt regulatory links between unicellular and multicellular genes in cancer. Elife 8: pii: e40947, 2019

10. Casás-Selves M and Degregori J: How cancer shapes evolution, and how evolution shapes cancer. Evolution (N Y) 4: 624-634, 2011. 
11. Pruitt KD, Tatusova $\mathrm{T}$ and Maglott DR: NCBI reference sequence (RefSeq): A curated nonredundant sequence database of genomes, transcripts and proteins. Nucleic Acids Res 33 (Database Issue): D501-D504, 2005.

12. Sever R and Brugge JS: Signal transduction in cancer. Cold Spring Harb Perspect Med 5: a006098, 2015.

13. Lodish H, Berk A, Zipursky SL, Matsudaira P, Baltimore D and Darnell J: Oncogenic mutations affecting cell proliferation. In: Molecular Cell Biology. 4th edition. W. H. Freeman, New York, NY, 2000.

14. Vogelstein B, Papadopoulos N, Velculescu VE, Zhou S, Diaz LA Jr and Kinzler KW: Cancer genome landscapes. Science 339: 1546-1558, 2013.

15. Miller MA: Driver mutations take the wheel in invasive yet nonmalignant disease. Sci Transl Med 9: pii: eaan8194, 2017.

16. McFarland CD, Yaglom JA, Wojtkowiak JW, Scott JG, Morse DL, Sherman MY and Mirny LA: The damaging effect of passenger mutations on cancer progression. Cancer Res 77: 4763-4772, 2017.

17. McFarland CD, Korolev KS, Kryukov GV, Sunyaev SR and Mirny LA: Impact of deleterious passenger mutations on cancer progression. Proc Natl Acad Sci USA 110: 2910-2915, 2013.

18. Chen J, Sun M and Shen B: Deciphering oncogenic drivers: From single genes to integrated pathways. Brief Bioinform 16 : 413-1428, 2015.

19. Zhang J and Zhang S: Discovery of cancer common and specific driver gene sets. Nucleic Acids Res 45: e86, 2017.

20. Vogelstein B and Kinzler KW: Cancer genes and the pathways they control. Nat Med 10: 789-799, 2004.

21. Levine AJ: p53, the cellular gatekeeper for growth and division. Cell 88: 323-331, 1997.

22. Lu WJ, Amatruda JF and Abrams JM: p53 ancestry: Gazing through an evolutionary lens. Nat Rev Cancer 9: 758-762, 2009.

23. Jegga AG, Inga A, Menendez D, Aronow BJ and Resnick MA: Functional evolution of the p53 regulatory network through its target response elements. Proc Natl Acad Sci USA 105: 944-949, 2008

24. Belyi VA, Ak P, Markert E, Wang H, Hu W, Puzio-Kuter A and Levine AJ: The origins and evolution of the p53 family of genes. Cold Spring Harb Perspect Biol 2: a001198, 2010.

25. Levine AJ and Oren M: The first 30 years of p53: Growing ever more complex. Nat Rev Cancer 9: 749-758, 2009

26. Joerger AC and Fersht AR: The p53 pathway: Origins, inactivation in cancer, and emerging therapeutic approaches. Annu Rev Biochem 85: 375-404, 2016.

27. Fresno Vara JA, Casado E, de Castro J, Cejas P, Belda-Iniesta C and González-Barón M: PI3K/Akt signalling pathway and cancer. Cancer Treat Rev 30: 193-204, 2004.

28. Philippon $\mathrm{H}$, Brochier-Armanet $\mathrm{C}$ and Perrière G: Evolutionary history of phosphatidylinositol-3-kinases: Ancestral origin in eukaryotes and complex duplication patterns. BMC Evol Biol 15 226, 2015.

29. Kriplani N, Hermida MA, Brown ER and Leslie NR: Class I PI 3-kinases: Function and evolution. Adv Biol Regul 59: 53-64, 2015.

30. Bertucci MC and Mitchell CA: Phosphoinositide 3-kinase and INPP4B in human breast cancer. Ann N Y Acad Sci 1280: 1-5, 2013.

31. LoPiccolo J, Blumenthal GM, Bernstein WB and Dennis PA: Targeting the PI3K/Akt/mTOR pathway: Effective combinations and clinical considerations. Drug Resist Updat 11: 32-50, 2008

32. Park S, Chapuis N, Tamburini J, Bardet V, Cornillet-Lefebvre P, Willems L, Green A, Mayeux P, Lacombe C and Bouscary D: Role of the PI3K/AKT and mTOR signaling pathways in acute myeloid leukemia. Haematologica 95: 819-828, 2010.

33. Polychronidou E, Vlachakis D, Vlamos P, Baumann $\mathrm{M}$ and Kossida S: Notch signaling and ageing. Adv Exp Med Biol 822. 25-36, 2015

34. Li L, Tang P, Li S, Qin X, Yang H, Wu C and Liu Y: Notch signaling pathway networks in cancer metastasis: A new target for cancer therapy. Med Oncol 34: 180, 2017.

35. Kwon OJ, Zhang L, Wang J, Su Q, Feng Q, Zhang XH, Mani SA, Paulter R, Creighton CJ, Ittmann MM and Xin L: Notch promotes tumor metastasis in a prostate-specific Pten-null mouse model J Clin Invest 126: 2626-2641, 2016.

36. Weng AP, Ferrando AA, Lee W, Morris JP IV, Silverman LB, Sanchez-Irizarry C, Blacklow SC, Look AT and Aster JC: Activating mutations of NOTCH1 in human T cell acute lymphoblastic leukemia. Science 306: 269-271, 2004.

37. Lobry C, Oh P, Mansour MR, Look AT and Aifantis I: Notch signaling: Switching an oncogene to a tumor suppressor. Blood 123: 2451-2459, 2014.
38. Mosedale G, Niedzwiedz W, Alpi A, Perrina F, Pereira-Leal JB, Johnson M, Langevin F, Pace P and Patel KJ: The vertebrate Hef ortholog is a component of the Fanconi anemia tumor-suppressor pathway. Nat Struct Mol Biol 12: 763-771, 2005.

39. Murphy ME: The HSP70 family and cancer. Carcinogenesis 34 $1181-1188,2013$

40. Rosenzweig R, Nillegoda NB, Mayer MP and Bukau B: The Hsp70 chaperone network. Nat Rev Mol Cell Biol 20: 665-680, 2019.

41. Calderwood SK and Gong J: Molecular chaperones in mammary cancer growth and breast tumor therapy. J Cell Biochem 113: 1096-1103, 2012.

42. Vander Heiden MG, Cantley LC and Thompson CB Understanding the Warburg effect: The metabolic requirements of cell proliferation. Science 324: 1029-1033, 2009.

43. Warburg O: On the origin of cancer cells. Science 123: 309-314, 1956.

44. Lunt SY and Vander Heiden MG: Aerobic glycolysis: Meeting the metabolic requirements of cell proliferation. Annu Rev Cell Dev Biol 27: 441-464, 2011

45. Alfarouk KO, Verduzco D, Rauch C, Muddathir AK, Bashir Adil HH, Elhassan GO, Ibrahim ME, David Polo Orozco J, Cardone RA, Reshkin SJ and Harguindey S: Glycolysis, tumor metabolism, cancer growth and dissemination. A new pH-based etiopathogenic perspective and therapeutic approach to an old cancer question. Oncoscience 1: 777-802, 2014

46. DeBerardinis RJ and Chandel NS: Fundamentals of cancer metabolism. Sci Adv 2: e1600200, 2016.

47. Dang CV: Links between metabolism and cancer. Genes Dev 26 877-890, 2012

48. Phan LM, Yeung SC and Lee MH: Cancer metabolic reprogramming: Importance, main features, and potentials for precise targeted anti-cancer therapies. Cancer Biol Med 11: 1-19, 2014.

49. Stine ZE, Walton ZE, Altman BJ, Hsieh AL and Dang CV: MYC, metabolism, and cancer. Cancer Discov 5: 1024-1039, 2015.

50. Halestrap AP: The monocarboxylate transporter family-Structure and functional characterization. IUBMB Life 64: 1-9, 2012.

51. Baltazar F, Pinheiro C, Morais-Santos F, Azevedo-Silva J, Queirós O, Preto A and Casal M: Monocarboxylate transporters as targets and mediators in cancer therapy response. Histol Histopathol 29: 1511-1524, 2014.

52. Perez-Escuredo J, Van Hée VF, Sboarina M, Falces J, Payen VL, Pellerin L and Sonveaux P: Monocarboxylate transporters in the brain and in cancer. Biochim Biophys Acta 1863: 2481-2497, 2016.

53. Ullah MS, Davies AJ and Halestrap AP: The plasma membrane lactate transporter MCT4, but not MCT1, is up-regulated by hypoxia through a HIF-1alpha-dependent mechanism. J Biol Chem 281: 9030-9037, 2006

54. San-Millan I and Brooks GA: Reexamining cancer metabolism: Lactate production for carcinogenesis could be the purpose and explanation of the Warburg Effect. Carcinogenesis 38: 119-133, 2017.

55. Payen VL, Mina E, Van Hée VF, Porporato PE and Sonveaux P: Monocarboxylate transporters in cancer. Mol Metab 33: 48-66, 2020.

56. Martinez-Outschoorn UE, Curry JM, Ko YH, Lin Z, Tuluc M, Cognetti D, Birbe RC, Pribitkin E, Bombonati A, Pestell RG, et al: Oncogenes and inflammation rewire host energy metabolism in the tumor microenvironment: RAS and $\mathrm{NF} \kappa \mathrm{B}$ target stromal MCT4. Cell Cycle 12: 2580-2597, 2013.

57. Bovenzi CD, Hamilton J, Tassone P, Johnson J, Cognetti DM, Luginbuhl A, Keane WM, Zhan T, Tuluc M, Bar-Ad V, et al: Prognostic indications of elevated MCT4 and CD147 across cancer types: A Meta-analysis. Biomed Res Int 2015: 242437, 2015.

58. Pavlova NN and Thompson CB: The emerging hallmarks of cancer metabolism. Cell Metab 23: 27-47, 2016.

59. Wahlstrom $T$ and Henriksson MA: Impact of MYC in regulation of tumor cell metabolism. Biochim Biophys Acta 1849: 563-569, 2015.

60. Bott JA, Peng IC, Fan Y, Faubert B, Zhao L, Li J, Neidler S, Sun Y, Jaber N, Krokowski D, et al: Oncogenic Myc induces expression of glutamine synthetase through promoter demethylation. Cell Metab 22: 1068-1077, 2015.

61. Kumada Y, Benson DR, Hillemann D, Hosted TJ, Rochefort DA, Thompson CJ, Wohlleben W and Tateno Y: Evolution of the glutamine synthetase gene, one of the oldest existing and functioning genes. Proc Natl Acad Sci USA 90: 3009-3013, 1993. 
62. Hill RT, Parker JR, Goodman HJ, Jones DT and Woods DR Molecular analysis of a nove glutamine synthetase of the anaerobe Bacteroides fragilis. J Gen Microb 135: 3271-3279, 1989.

63. Goodman HJ and Woods DR: Cloning and nucleotide sequence of the Butyrivibrio fibrisolvens gene encoding a type III glutamine synthetase. J Gen Micro 139: 1487-1493, 1993.

64. Pesole G, Gissi C, Lanave C and Saccone C: Glutamine synthetase gene evolution in bacteria. Mol Biol Evol 12: 189-197, 1995.

65. Shatters RG and Kahn JL: Glutamine synthetase II in Rhizobium: Reexamination of the proposed horizontal transfer of DNA from eukaryotes to prokaryotes. J Mol Evol 2: 422-428, 1989.

66. Lee TI and Young RA: Transcriptional regulation and its misregulation in disease. Cell 152: 1237-1251, 2013.

67. Werner F and Grohmann D: Evolution of multi-subunit RNA polymerases in the three domains of life. Nat Rev Microbiol 9: 85-98, 2011.

68. Shin DS, Pratt AJ and Tainer JA: Archaeal genome guardians give insights into eukaryotic DNA replication and damage response proteins. Archaea 2014: 206735, 2014.

69. Warner JR: The economics of ribosome biosynthesis in yeast. Trends Biochem Sci 24: 437-440, 1999.

70. Drygin D, Rice WG and Grummt I: The RNA polymerase I transcription machinery: An emerging target for the treatment of cancer. Annu Rev Pharmacol Toxicol 50: 131-156, 2010.

71. Arabi A, Wu S, Ridderstråle K, Bierhoff H, Shiue C, Fatyol K, Fahlén S, Hydbring P, Söderberg O, Grummt I, et al: c-Myc associates with ribosomal DNA and activates RNA polymerase I transcription. Nat Cell Biol 7: 303-310, 2005

72. Grandori C, Gomez-Roman N, Felton-Edkins ZA, Ngouenet C, Galloway DA, Eisenman RN and White RJ: c-Myc binds to human ribosomal DNA and stimulates transcription of rRNA genes by RNA polymerase I. Nat Cell Biol 7: 311-318, 2005.

73. Mayer C and Grummt I: Ribosome biogenesis and cell growth: mTOR coordinates transcription by all three classes of nuclear RNA polymerases. Oncogene 25: 6384-6391, 2006.

74. Zhang C, Comai L and Johnson DL: PTEN represses RNA Polymerase I transcription by disrupting the SL1 complex. Mol Cell Biol 25: 6899-6911, 2005.

75. Grummt I: Life on a planet of its own: Regulation of RNA polymerase I transcription in the nucleolus. Genes Dev 17: 1691-1702, 2003.

76. Luo Z, Lin C, Guest E, Garrett AS, Mohaghegh N, Swanson S, Marshall S, Florens L, Washburn MP and Shilatifard A: The super elongation complex family of RNA polymerase II elongation factors: Gene target specificity and transcriptional output. Mol Cell Biol 32: 2608-2617, 2012.

77. Rahl PB, Lin CY, Seila AC, Flynn RA, McCuine S, Burge CB, Sharp PA and Young RA: c-Myc regulates transcriptional pause release. Cell 141: 432-445, 2010.

78. Smith E, Lin C and Shilatifard A: The super elongation complex (SEC) and MLL in development and disease. Genes Dev 25: 661-672, 2011

79. Aguilo F, Zhou MM and Walsh MJ: Long noncoding RNA, polycomb, and the ghosts haunting INK4b-ARF-INK4a expression. Cancer Res 71: 5365-5369, 2011.

80. Sliwoski G, Kothiwale S, Meiler J and Lowe EW Jr: Computational methods in drug discovery. Pharmacol Rev 66: 334-395, 2014.

81. Park H, Bahn YJ and Ryu SE: Structure-based de novo design and biochemical evaluation of novel Cdc25 phosphatase inhibitors. Bioorg Med Chem Lett 19: 4330-4334, 2009.
82. Vangrevelinghe E, Zimmermann K, Schoepfer J, Portmann R, Fabbro D and Furet P: Discovery of a potent and selective protein kinase CK2 inhibitor by high-throughput docking. J Med Chem 46: 2656-2662, 2003.

83. Kreeger PK and Lauffenburger DA: Cancer systems biology: A network modeling perspective. Carcinogenesis 31: 2-8, 2010.

84. San Lucas FA, Fowler J, Chang K, Kopetz S, Vilar E and Scheet P: Cancer in silico drug discovery: A systems biology tool for identifying candidate drugs to target specific molecular tumor subtypes. Mol Cancer Ther 13: 3230-3240, 2014.

85. Carter H, Chen S, Isik L, Tyekucheva S, Velculescu VE, Kinzler KW, Vogelstein B and Karchin R: Cancer-specific high-throughput annotation of somatic mutations: Computational prediction of driver missense mutations. Cancer Res 69: 6660-6667, 2009.

86. Chen W, Li Y and Wang Z: Evolution of oncogenic signatures of mutation hotspots in tyrosine kinases supports the atavistic hypothesis of cancer. Sci Rep 8: 8256, 2018

87. Trigos AS, Pearson RB, Papenfuss AT and Goode DL: Altered interactions between unicellular and multicellular genes drive hallmarks of transformation in a diverse range of solid tumors. Proc Natl Acad Sci USA 114: 6406-6411, 2017.

88. Zhou XH, Chu XY, Xue G, Xiong JH and Zhang HY: Identifying cancer prognostic modules by module network analysis. BMC Bioinformatics 20: 85, 2019.

89. DeGregori J: Evolved tumor suppression: Why are we so good at not getting cancer? Cancer Res 71: 3739-3744, 2011.

90. Dunning Hotopp JC: Horizontal gene transfer between bacteria and animals. Trends Genet 27: 157-163, 2011.

91. Robinson KM, Sieber KB and Dunning Hotopp JC: A review of bacteria-animal lateral gene transfer may inform our understanding of diseases like cancer. PLoS Genet 9: e1003877, 2013.

92. Baba Y, Iwatsuki M, Yoshida N, Watanabe M and Baba H: Review of the gut microbiome and esophageal cancer: Pathogenesis and potential clinical implications. Ann Gastroenterol Surg 1: 99-104, 2017.

93. Riley DR, Sieber KB, Robinson KM, White JR, Ganesan A, Nourbakhsh S and Dunning Hotopp JC: Bacteria-human somatic cell lateral gene transfer is enriched in cancer samples. PLoS Comput Biol 9: e1003107, 2013.

94. Cao Y: Tumorigenesis as a process of gradual loss of original cell identity and gain of properties of neural precursor/progenitor cells. Cell Biosci 7: 61, 2017.

95. Trigos AS, Pearson RB, Papenfuss AT and Goode DL: How the evolution of multicellularity set the stage for cancer. $\mathrm{Br} \mathrm{J}$ Cancer 118: 145-152, 2018.

96. Merlo LM, Pepper JW, Reid BJ and Maley CC: Cancer as an evolutionary and ecological process. Nat Rev Cancer 6: 924-935, 2006.

This work is licensed under a Creative Commons Attribution-NonCommercial-NoDerivatives 4.0 International (CC BY-NC-ND 4.0) License. 